\title{
Les sélectines et leur rôle dans l'interaction des leucocytes avec les cellules endothéliales
}

Les sélectines constituent une nouvelle famille de récepteurs d'adhérence des leucocytes à l'endothélium. Au nombre de trois, elles ont en commun un domaine extracellulaire $\mathbf{C}$-lectinique qui interagit spécifiquement avec un déterminant osidique sialyl Lewis ${ }^{x}$. Les sélectines $\mathbf{P}$ et $\mathbf{E}$ ne sont exprimées à la surface des cellules qu'après activation adéquate. La sélectine $\mathbf{P}$ est présente à la surface des cellules endothéliales et des plaquettes stimulées par la thrombine ou l'histamine, alors que la sélectine $\mathbf{E}$ n'est exprimée qu'à la surface des cellules endothéliales stimulées par l'IL-1, le TNF $\alpha$ et le LPS. Toutes les deux interagissent avec les neutrophiles et les monocytes. La sélectine $L$, enfin, a été découverte initialement sur les lymphocytes et participe à leur domiciliation dans les ganglions lymphatiques périphériques. Ces progrès récents dans la caractérisation du rôle des sélectines dans les réactions inflammatoires et leur spécificité pourraient déboucher sur la synthèse d'inhibiteurs oligosaccharidiques de l'inflammation.

\section{Geneviève Durand}

\section{ADRESSE ET TIRÉS À PART}

G. Durand : professeur des universités, praticien hospitalier. Laboratoire de biochimie générale, centre pharmaceutique de ChâtenayMalabry, rue J.-B. Clément, 92296 Châtenay-Malabry Cedex, France.

$\mathrm{m} / \mathrm{s} n^{\circ} 10$ vol. 8, décembre 92 'endothélium vasculaire, constitué d'une couche monocellulaire, est un tissu dynamique qui contrôle parmi divers processus le trafic des cellules entre le sang et les espaces extravasculaires. Si l'on considère globalement le devenir des polynucléaires et des monocytes circulants, il apparaît qu'il y a interaction physiologique permanente d'un petit nombre d'entre eux avec la paroi vasculaire, suivie de leur diapédèse entre les cellules endothéliales. Les polynucléaires n'ont pas de des- tinée particulière et sont progressivement éliminés de l'organisme, alors que les monocytes se différencient en macrophages tissulaires. Au cours d'une inflammation aiguë, le recrutement séquentiel des polynucléaires et des monocytes au site inflammatoire s'accroît considérablement par suite d'une meilleure efficacité de l'adhérence des cellules à l'endothélium. Ce phénomène d'adhérence a lieu principalement sur les cellules endothéliales des veinules postcapillaires. Dans ces veinules, le flux sanguin est lent, les forces de friction 


\section{RÉFÉRENCES}

1. Fischer A. Quatre familles de molécules responsables de l'adhérence intercellulaire. médecine/sciences $1991 ; 7$ : 540-2.

2. Degos L, Kahn A. Adhésion cellulaire. médecine/sciences $1987 ; 3$ : 314-5.

3. Margucrie G, Berthicr R, Duperray A, Hudry-Clergeon $G$, Uzan $G$. Les adhésines ccllulaires : variations sur un thème. médecine/sciences $1987 ; 3: 326-33$

4. Thiéry JP, Dufour S, Duband JL. Fibronectines, morphogenèse et migrations cellulaircs. médecine/sciences $1987 ; 3$ : 316-25.

5. Fischer A, Auffray C, Durandy A. Les molécules d'adhésion des lymphocytes T. médecine/sciences $1987 ; 3$ : 334-42.

6. Nécl D, Aubéry M, Derappc C. Glycobiologic et cellules sanguines. médecine/sciences $1992 ; 8$ : 233-8.

7. McEver RP. Sclectins : novel reccptors that mediate lcukocyte adhesion during inflammation. Thromb Haemost 1991; 65 : 223-8.

8. Drickamer K. Two distinct classes of carbohydrate-recognition domains in animal lectins. J Biol Chem 1988 ; 263 : 9557-60.

9. Lasky LA, Singer MS, Yednock TA, et al. Cloning of a lymphocyte homing receptor reveals a lectin domain. Cell $1989 ; 56$ : 1045-55.

10. Sicgelman $\mathrm{MH}$, Van de Rijn $\mathrm{M}$, Weissman IL. Mousc lymph node homing receptor cDNA clonc encodes a glycoprotein revealing tandem interaction domains. Science $1989 ; 243: 1165-72$

11. Tedder TF, Isaacs CM, Ernst TJ, et al. Isolation and chromosomal localization of cDNAs encoding a novel human lymphocytc ccll surface molecule, Lam-1. J Exp Med $1989 ; 170: 123-33$.

12. Bowen BR, Nguyen T, Lasky LA. Characterization of a human homologuc of the murine peripheral lymph node homing receptor. J Cell Biol 1989; 109 : 421-7.

13. Gallatin WM, Weissman IL, Butcher EC. A celle-surface molecule involved in organ-specific homing of lymphocytes. Nature $1983 ; 304: 30-4$

14. Lewinsohn DM, Bargatze RF, Butcher EC. Leukocytc-endothelial cell recognition : evidence of a common molecular mechanism shared by neutrophils, lymphocytes, and other lcukocytes. J Immunol $1987 ; 138$ : 4313-21.

15. Johnston GI, Cook RG, McEver RP. Cloning of GMP-140 a granule membranc protein of platelets and endothclium : sequence similarity to protcins involved in cell adhesion and inflammation. Cell 1989 ; $56: 1033-44$ auxquelles sont soumises les cellules sont faibles, ce qui favorise leur adhérence. Au cours de l'inflammation, les forces d'adhérence augmentent par une expression massive de protéines d'adhérence à la surface de l'endothélium, et la diapédèse est grandement favorisée.

Les lymphocytes se comportent différemment en ce sens qu'ils circulent du sang vers les tissus lymphoïdes ct/ou les sites d'inflammation pour revenir ensuite dans la circulation sanguine après passage à travers les vaisseaux lymphatiques, les ganglions lymphatiques et le canal thoracique. Ils adhèrent à l'cndothélium des veinules post-capillaires des organes lymphoïdes secondaires (ganglions lymphatiques centraux et périphériques, plaques de Peycr) et des sites d'inflammation chronique. Les ccllules endothéliales de ces régions sont volumineuses et cubiques, comparées à l'endothélium mince et plat généralement observé dans le système microcirculatoire, et sont à l'origine du terme high endothelium venules donné à ces veinules spécialisées. Cette différence morphologique s'accompagne d'une différence fonctionnelle avec notamment l'expression à leur surface apicale de molécules d'adhérence impliquées dans la domiciliation (homing) de divers lymphocytes qui par migration trans-cellulaire atteignent leur site d'action spécifique.

La première étape de la migration trans-endothélialc des leucocytes consiste donc en une adhérence hétérophile qui met en jeu trois familles de molécules [1] : les protéines d'adhérence apparentées aux immunoglobulines, les intégrines et les sélectines. Les protéines ICAM-1 et -2 (intercellular adhesion molecules) et la VCAM-1 (vascular cell adhesion molecule) possèdent dans la partie extracellulaire de leur structure des domaines analogues à ccux constituant les chaînes lourdes et légères des immunoglobulines. Les intégrines sont des hétérodimères résultant d'une association de chaînes $\alpha$ et $\beta$ [2-4]. Les neutrophiles et les monocytes expriment des $\beta 2$ intégrines de trois types selon la nature de la chaîne $\alpha$ (CD11a/CD18, CD11b/CD18, CD11c/CD18) qui interagissent avec les ICAM-1 et -2 exprimées à la surface des ccllules endothćliales. Ces $\beta 2$ intégrines sont reconnues également par d'autres ligands : le C3 bi pour CD11c/18 ; le $\mathrm{C} 3 \mathrm{bi}$, le fibrinogène et le facteur $\mathrm{X}$ pour CD11b/18. Les lymphocytes expriment LFA-1 (lymphocyte function associated antigen-1) qui est une $\beta 2$ intégrine (CD11a/CD18) qui interagit avec les ICAM, et VLA-4 (very late antigen-4) qui est une $\beta 1$ intégrine (CD49d/CD29) ayant comme ligands le fibrinogène et la VCAM-1 [5]. Enfin, la dernière famille est constituée par les sélectines $[6,7]$. Ce nom provient d'une forte analogie structurale avec des récepteurs transmembranaires regroupés par Drickamer [8] sous le terme de C-lectines. Leur ligand est de nature osidique.

\section{La famille des sélectines}

Le terme de sélectine suivi d'une lettre majuscule indiquant le type cellulaire dans lequel elles ont été initialement isolées sera utilisé dans la suite de l'article, les synonymes étant rappelés dans le Tableau $I$. La sélectine L est exprimée constitutivement à la surface des lymphocytes murins $[9,10]$ et humains $[11,12]$. Ces sélectines murine et humaine sont reconnues par le même anticorps et ont la même fonction en intervenant spécifiquement dans la migration de lymphocytes vers les ganglions lymphatiques périphériques [13]. La sélectine $\mathrm{L}$ est aussi présente à la surface des neutrophiles et des monocytes humains [14]. La sélectine $P$ humaine présente dans les granules $\alpha$ des plaquettes et les corps de WeiberPalade des cellules endothéliales est exprimée à leur surface après activation [15]. La sélectine E n'apparaît à la surface des ccllules endothéliales humaines qu'après activation; il s'agit d'une synthèse de novo [16]. Les gènes de ces différentes sélectines ont été clonés et séquencés la même année, en 1989 [9-13, 15, 16].

Ces différentes molécules sont des glycoprotéines transmembranaires de type 1 (extrémité $\mathrm{N}$-terminale extracellulaire) dont la séquence peptidique a été déduite de l'analyse des nucléotides des ADNc. Après le peptide signal hydrophobe, se succèdent une très longue séquence riche en sites potentiels de N-glycosylation (asn-Xthr/sér) qui constitue la partie extracellulaire, puis une courte séquence 


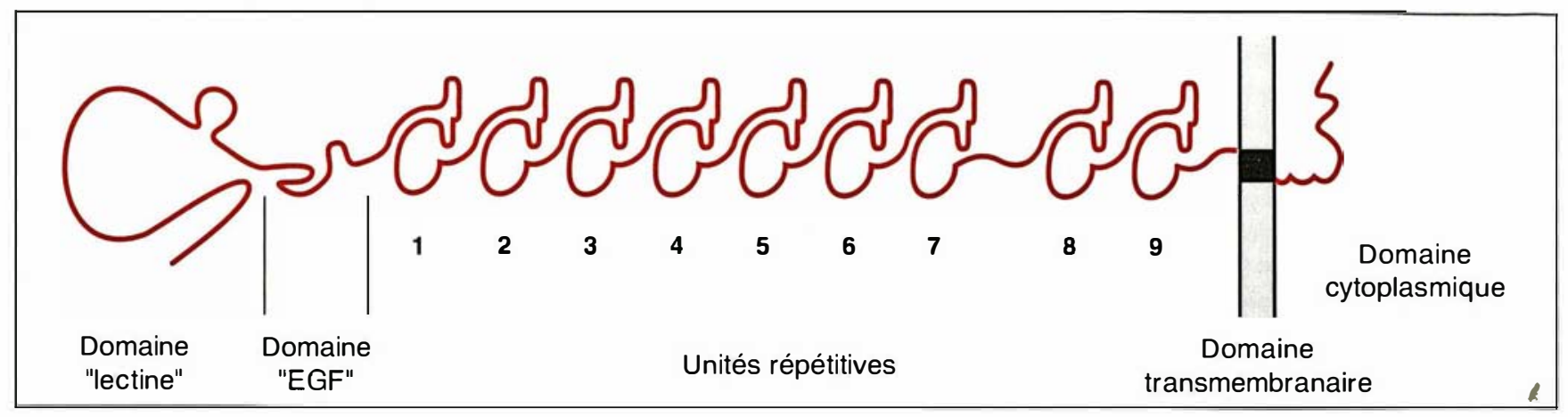

Figure 1. La famille des sélectines. Représentation schématique de la structure de la sélectine P. (D'après [15].).

hydrophobe qui, suivie de quelques acides aminés basiques, représente le domaine membranaire, et enfin la courte extrémité C-terminale cytoplasmique.

La partie extracellulaire est organisée en trois domaines (figure 1). L'extrćmité $\mathrm{N}$-terminale est constituée par le domaine C-lectinique, qui comporte environ 130 acides aminés dont quatre résidus cystéine et 15 à 20 acides aminés communs à l'ensemble des Clectines. L'importance de ces acides aminés dans la reconnaissance de résidus osidyles spécifiques a pu être démontrée par des ćtudes de mutagenèse dirigée. Cette interaction dépend du calcium. Le domaine suivant est court (environ 30 acides aminés) et présente une grande analogie avec le facteur de croissance de l'épiderme (EGF) (16 résidus cystéine et trois résidus glycine). Le domaine suivant, très long, est constitué d'unités répétitives en tandem comportant chacune six résidus cystéine, rappelant les séquences communes à certaines protéines contrôlant le complément. Les structures des trois sćlec- tines présentent $60 \%$ d'identité dans les domaines lectiniques et EGF, et environ $40 \%$ dans les unités répétitives. Ces analogies structurales avec des molécules connues et leur rôle dans l'adhćrence sont à l'origine de l'acronyme qui leur est aussi affecté LEC-CAM. (L pour lectine, $\mathrm{E}$ pour EGF et $\mathrm{C}$ pour complément).

Si le domaine C-lectinique intervient bien dans la liaison avec le type cellulaire approprié, il semble que le domaine EGF soit également nécessaire à cette interaction [17, 18], sans que l'on sache toutefois s'il s'agit d'une participation directe ou indirecte en maintenant par exemple une conformation spatiale correcte.

\section{Nature osidique des ligands}

Les nombreuses approches méthodologiques pour définir la nature du ligand reposent sur la notion que ce ligand est de nature osidique. Un premier type de protocoles consiste à mettre en contact la cellule exprimant le ligand avec la sélectine, soit puri- fiée et fixée sur un support inerte, soit présente à la surface de cellules transfectées par l'ADNc correspondant. On peut alors déterminer la nature du saccharide impliqué dans cette interaction par des études d'inhibition en utilisant des inhibiteurs osidiques ou des anticorps monoclonaux dirigés spécifiquement contre des structures osidiques déterminées [19]. On peut aussi recourir à l'action d'exoglycosidases qui, en éliminant séquentiellement les résidus osidyles, permettent d'établir la structure minimale indispensable à l'interaction. Cette diversité de méthodologie explique en partie certains résultats contradictoires. Un autre type d'étude consiste à exprimer à la surface d'une cellule un résidu osidyle et à déterminer l'incidence de la présence de ce résidu sur l'interaction étudiée. L'addition d'un résidu osidyle sur un glycoconjugué (glycolipide ou glycoprotéine) ne peut se faire que par transfection d'un ADNc codant pour une glycosyltransférase dans une cellule qui n'exprime pas constitutivement cette enzyme. Ainsi

Tableau I

DIFFÉRENTES DÉNOMINATIONS DES SÉLECTIVES

\begin{tabular}{|c|c|c|c|}
\hline \multicolumn{2}{|c|}{ Sélectine L } & Sélectine $\mathbf{P}$ & Sélectine E \\
\hline TQ1 & LAM-1 & GMP-140 & ELAM-1 \\
\hline DREG. 56 & LEU-8 & PADGEM & \\
\hline MEL 14 & LECAM-I & CD62 & \\
\hline gp 9OMEL & & LECAM-3 & \\
\hline
\end{tabular}




\section{RÉFÉRENCES}

16. Bevilacqua MP, Stengelin S, Gimbrone $\mathrm{MA}$, et al. Endothelial leukocyte adhesion molecule 1: an inducible receptor for neutrophils related to complement regulatory proteins and lectins. Science 1989; 243 $1160-5$.

1

17. Walz G, Aruffo A, Kolanus W, et al. Recognition by ELAM-1 of the sialyl-Le determinant on myeloid and tumor cells. Science $1990 ; 250$ : 1132-45.

18. Bowen BR, Fennie C, Lasky LA. The MEL-14 antibody binds to the lectin domain of the murine peripheral lymph node homing receptor. J Cell Biol 1990; 110 : 147-53.

19. Brandley BK, Swiedler SJ, Robbins PW. Carbohydrate ligands of the LEC ccl adhesion molecules. Cell 1990 ; 63 : 861-3.

20. Goelz SE, Hession C, Goff D, et al ELFT : a gene that directs the expression of an ELAM-1 ligand. Cell 1990; 63 : 1349-56.

21. Lowe JB, Stoolman LM, Nair RP, e al. ELAM-1. Dependant cell adhesion to vascular endothelium determined by a trans fected human fucosyltransferase cDNA. Cell 1990 ; $63: 475-84$.

22. Lowe JB, Kukowska-Latallo JF, Nair $\mathrm{RP}$, et al. Molccular cloning of a human fucosyltransferase gene that determines expression of the Lewis $x$ and VIM-2 epitopes but not ELAM-1 dependant cell adhesion. J Biol Chem 1991; 266 : 17467-77.

23. Philipps ML, Nudelman E, Gaeta FCA, et al. ELAM-1 mediates cell adhesion by recognition of a carbohydrate ligand sialyl-Le ${ }^{\mathrm{x}}$. Science $1990 ; 250$ : 1130-2.

24. Handa K, Nudelman ED, Stroud MR, et al. Selectins GMP-140 (CD62; PADGEM) binds to sialosyl-Le ${ }^{\mathrm{a}}$ and sialosylLe $^{\times}$, and sulfated glycans modulate this binding. Biochem Biophys Res Commun 1991 ; 181: 1223-30

25. Polley MJ, Phillips ML, Wayner E, et al. CD62 and endothelial cell-leukocyte adhesion molecule 1 (ELAM-1) recognize the same carbohydrate ligand, sialyl-Lewis x. Proc Natl Acad Sci USA 1991 ; 88 : 6224-8.

26. Berg EL, Robinson MK, Mansson O, et al. A carbohydrate domain common to both sialyl $\mathrm{Le}^{\mathrm{a}}$ and sialyl $\mathrm{Le}^{x}$ is recognized by the endothelial cell leukocyte adhesion molecule ELAM-1.J Biol Chem 1991 ; $266: 14869-72$.

27. Larsen GR, Sako D, Ahern TJ, et al. P-selectin and E-selectin. Distinct but overlapping leukocyte ligand specificities. $J$ Biol Chem 1992; 267 : 11104-10. la transfection d'un fragment d'ADN génomique codant pour une $\alpha$ fucosyltransférase dans les cellules $\mathrm{COS}$ et $\mathrm{CHO}$ a permis de mettre en évidence l'importance du résidu fucosyle du déterminant sialyl Lewis $^{\times}\left(\mathrm{SLe}^{\times}\right)$dans l'interaction avec la sélectine $\mathrm{E}[6,20-22]$ et de démontrer que le ligand de la sélectine E était $\mathrm{SLe}^{\times}$et en aucun cas les structures Le ${ }^{\times}$et VIM2 (Tableau II) [21]. Les sélectines $P$ et $E$ reconnaissent les mêmes déterminants antigéniques $\mathrm{SLe}^{\times}$et SLe ${ }^{\text {i }}$ (Tableau II) portés indifféremment par des glycoprotéines, des mucines ou des glycolipides $[17,23-26]$. Il semble toutefois que la spécificité de la sélectine $\mathrm{P}$ dépende d'autres composés protéiques et/ou osidiques [27] Quelle est, en fait, la nature exacte de la molécule qui, présente à la surface des neutrophiles, interagit avec la sélectine E des cellules endothéliales activées? Une seule étude montre qu'il s'agit majoritairement (40 à $60 \%$ ) de la sélectine L [28] qui, dans les neutrophiles, acquiert le déterminant $\mathrm{SLe}^{\times}$non détectable sur la sélectine L des lymphocytes. Certains auteurs ont toutefois montré que la sélectine $\mathrm{E}$ pouvait interagir avec certaines sous-populations de lymphocytes.

Dans les études portant sur la domi- ciliation des lymphocytes, les efforts ont porté en tout premier lieu sur la nature de la molécule qui, dans les ganglions lymphatiques périphériques, reconnaît la sélectine L. Il s'agit chez l'homme [29] et chez la souris [30] d'une glycoprotéine respectivement de 105 et $50 \mathrm{kDa}$. La protéine murine, qui présente une structure de type mucine avec deux domaines riches en résidus sérine et thréonine, est spécifiquement exprimée dans les high endothelial venules des ganglions lymphathiques périphériques [30]. Simultanément, il a été montré que le ligand osidique était aussi le déterminant SLe ${ }^{\times}$[31].

\section{Expression cellulaire des sélectines et de leur ligand}

L'utilisation d'anticorps bloquant spécifiquement l'interaction entre les cellules exprimant à leur surface les sélectines et un type cellulaire donné, a permis de définir le ou les type(s) cellulaire(s) impliqué(s) dans la reconnaissance. La sélectine L est constitutivement exprimée à la surface d'environ $70 \%$ des lymphocytes circulants qui interagissent avec les cellules endothéliales des ganglions lymphatiques périphériques, à l'exclu-

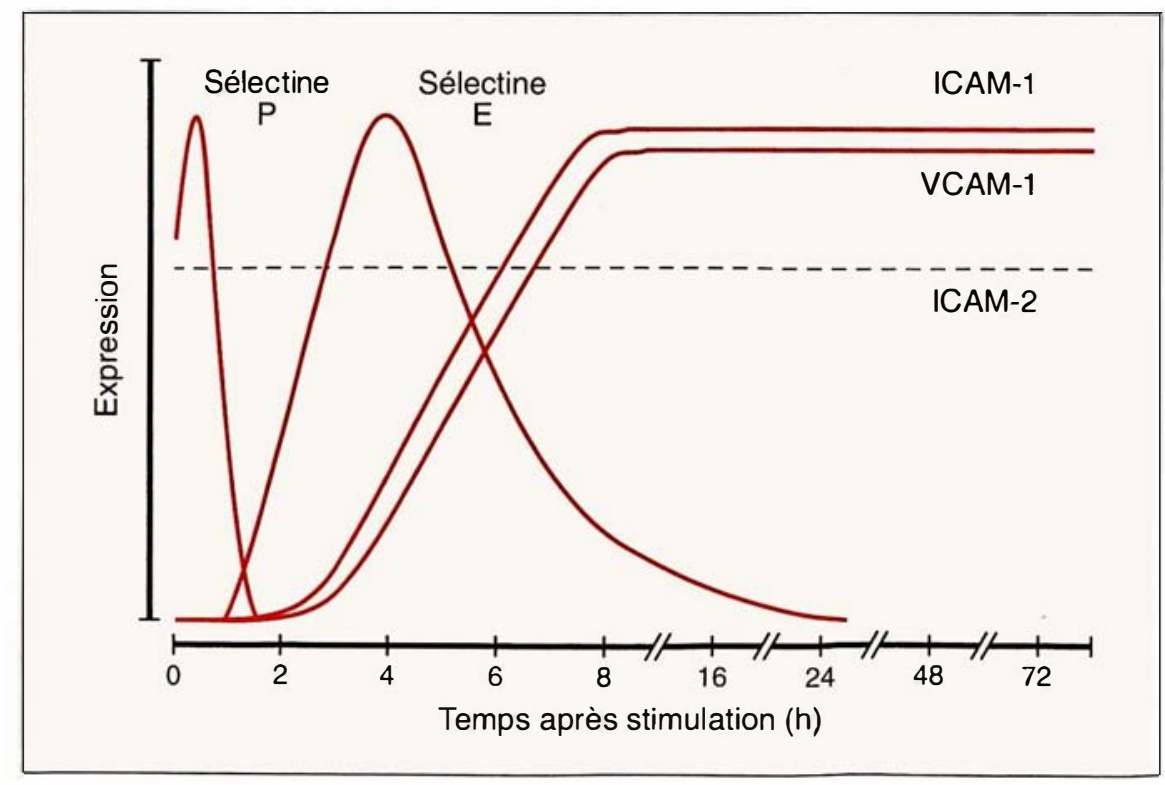

Figure 2. Expression séquentielle des molécules d'adhérence aux leucocytes, à la surface des cellules endothéliales activées. ( $D^{\prime}$ après [7].) 
STRUCTURES OLIGOSACCHARIDIQUES EXPRIMÉES À LA SURFACE DES LEUCOCYTES

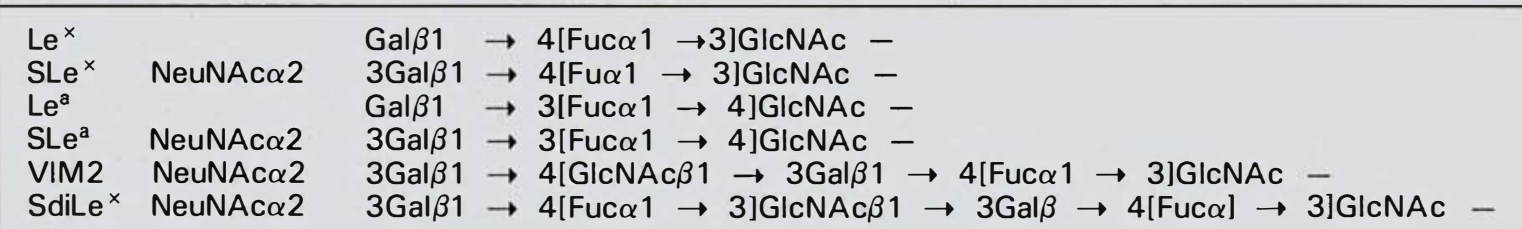

sion des autres tissus lymphoïdes. Elle peut interagir aussi avec l'endothélium de tissus présentant une inflammation chronique. Exprimée aussi sur les neutrophiles et les monocytes, elle interagit également avec les cellules endothéliales stimulées par le tumor necrosis factor $(\mathrm{TNF} \alpha)$, l'interleukine 1 $\beta$ (IL-1 $\beta$ ) et le lipopolysaccharide (LPS). Lorsque les leucocytes sont à leur tour activés, la sélectine $\mathrm{L}$ est rapidement relarguée par protéolyse dans le plasma, où elle est d'ailleurs détectée à une concentration de l'ordre du $\mu \mathrm{g} / \mathrm{ml}$.

La sélectine $P$ est présente, rappelons-le, dans les granules des plaquettes et des cellules endothéliales des veinules post-capillaires [32]. Le thrombine et l'histamine provoquent en quelques minutes et transitoirement l'expression de la sélectine $\mathrm{P}$ à la surface de ces cellules qui peuvent alors interagir avec les neutrophiles et les monocytes. La sélectine $\mathrm{P}$ disparaît par endocytose, 20 à 60 minutes après le stimulus [33].

La sélectine $\mathrm{E}$ n'est exprimée à la surface des mêmes cellules endothéliales qu'après activation par le TNF$\alpha$, l'IL1- $\beta$ et le LPS [34]. Le niveau d'expression est maximal entre 4 et 6 heures et diminue progressivement en 12 à 24 heures; cette protéine n'est détectée que sur les veinules post-capillaires. La sélectine E a également un rôle d'activateur, en induisant sur les neutrophiles la synthèse de $\beta 2$ intégrines [35]. Aucune de ces deux sélectines $\mathrm{E}$ et $\mathrm{P}$ n'est présente sur les lymphocytes.

\section{Relation avec d'autres molécules d'adhérence}

La migration des leucocytes aux sites d'inflammation fait intervenir d'autres molécules d'adhérence [1], 36]. Dans le défaut d'adhérence des $\mathrm{m} / \mathrm{s} n^{\circ} 10$ vol. 8, décembre 92 leucocytes dû à une absence de synthèse de la chaîne $\beta 2$, il n'y a pas de cellules phagocytaircs au site inflammatoire, ce qui démontre l'importance des $\beta 2$ intégrines dans la migration des leucocytes [5]. Ces $\beta 2$ intégrines interagissent avec des récepteurs endothéliaux de la superfamille des immunoglobulines, les ICAM. Alors qu'ICAM-2 est constitutivement exprimée à la surface des cellules endothéliales, ICAM-1 n'apparaît que lors d'une inflammation. Les lymphocytes qui migrent dans les tissus infectés, même dans les défauts d'adhérence leucocytaire, présentent eux aussi à leur surface des intégrines qui interagissent spécifiquement avec les différentes molécules d'adhérence des cellules endothéliales [7].

A u cours de l'inflammation, l'expression des $\beta 2$ intégrines des cellules phagocytaires est considérablement accrue par des agents synthétisés par divers types cellulaires proches du site infectieux, dont les cellules endothéliales : le facteur d'activation des plaquettes (PAF), l'interleukine 8 (IL-8) et la sélectine E. L'activation par la thrombine et l'histamine des cellules endothéliales conduit en quelques minutes à l'expression de PAF membranaire qui, à son tour, stimule l'expression des $\beta 2$ intégrines des neutrophiles. L'IL- 1 , le TNF- $\alpha$ et le LPS contrôlent la sécrétion de l'IL-8 et de la sélectine $E$ qui elles aussi stimulent la synthèse des $\beta 2$ intégrines, avec, en plus, des propriétés chimiotactiques.

Les différentes phases qui se déroulent à la surface des ccllules endothéliales et qui conduisent à l'adhérence des leucocytes à lcur surface peuvent être ainsi résumées (figure 2) : expression rapide et transitoire de la sélectine $\mathrm{P}$, expression différée de quelques heures et plus durable de la sélectine $\mathrm{E}$, expression soutenue d'ICAM-I et de VCAM-1 (ICAM-2 est maintenue à un taux basal). Simultanćment, le PAF et l'IL-8 produits séquentiellement par les cellules endothéliales activent les neutrophiles et induisent à leur surface l'expression de certaines intégrines. On peut admettre que, par le biais de la sélectinc E qui est expriméc sur les cellules endothéliales activées des veinules post-capillaires, les neutrophiles " roulent " sur l'endothélium (rolling). Puis, après activation des $\beta 2$ intégrines des neutrophiles qui interagissent avec les ICAM, cet effet cesse, l'adhérence se renforce et est suivic d'une migration entre les jonctions des cellules endothéliales. Dans un modèle d'étude in vitro et in vivo, il a étć montré que les $\beta 2$ intégrines n'interviennent dans l'adhérence que s'il y a eu contact préalable avec la sćlectine E [37, 38].

La sćlectine L des lymphocytes semble avoir un rôle essentiellement limité à leur passage dans les ganglions lymphatiques périphériques. Le trafic dans d'autres tissus fait appel à d'autres paires de molécules qui ne sont pas encore parfaitement identifiées.

Les progrès extrêmement rapides réalisés en trois ans dans l'étude de ces sélectines ouvrent de nouvelles perspectives en thérapeutique [6, 39]. Compte tenu de la nature osidique bien établie du ligand des sélectines $\mathrm{P}$ et $\mathrm{E}$ qui agissent au tout début du processus d'adhérence lors d'une inflammation locale, on peut imaginer de développer de nouveaux antiinflammatoires constitués d'oligosaccharides spécifiques de ces deux sélectines, à condition qu'ils ne viennent pas perturber des phénomènes d'adhérence physiologiquement vitaux, comme par exemple la circulation des lymphocytes 


\section{RÉFÉRENCES}

28. Picker LJ, Warnock A, Burns AR, et al. The neutrophil sclectin LECAM-1 presents carbohydrate ligands to the vascular selectins ELAM-1 and GMP-140. Cell $1991 ; 66: 921-33$

29. Berg EL, Robinson MK, Warnock RA, et al.. The human peripheral lymph node vascular addressin is a ligand for LECAM-1, the peripheral lymph node homing reccptor. J Cell Biol 1991; 114 : 343-9.

30. Lasky LA, Singer MS, Dowbenko D, et al. An cndothelial ligand for L-selectin is a novel mucin-like molcculc. Cell 1992 ; 69 : 927-38.

31. Foxall X, Watson SR, Dowbenko D, et al. The thrce members of the sclectins receptor family recognize a common carbohydrate cpitopc, the sialyl Lewis ${ }^{\times}$oligosaccharide. $J$ Cell Biol 1992 ; 117 : 895-902.

32. Larsen E, Ccli A, Gilbert GE, et al. PADGEM protcin : a receptor that mediates the interaction of activated platelets with neutrophils and monocytes. Cell 1989 ; 59 305-12.

33. Hattori R, Hamilton KK, Fugatc RD, et al. Stimulated secretion of endothelial von Willebrand factor is accompagnicd by rapid redistribution to the ccll surface of the intraccllular granulc membranc protein GMP-140. J Biol Chem 1989 ; 264 : 7768-71. 34. Bevilacqua MP, Pober JS, Mendrick $\mathrm{DL}$, et al. Identification of an inducible cndothclial-lcukocyte adhesion molcculc. Proc Natl Acad Sci USA 1987 ; 84 : 9238-42. 35. Lo SK, Lce S, Ramos RA, et al. Endothclial-leukocyte adhesion moleculc 1 stimulates the adhesive activity of leukocyte integrin CR3 (CD 11b/CD18, Mac-1 $\alpha \mathrm{m} \beta 2$ ) on human ncutrophils. J Exp Med $1991 ; 173: 1493-500$

36. Zimmerman GA, Prescott SM McIntyrc TM. Endothelial cell interactions whith granulocytes : tethering and signaling molcculcs. Immunol Today 1992; 13 93-100

37. Lawrence MB, Springer TA. Lcukocytes roll on a sclectin at physiologic flow rates. Distinction from and prercquisite for adhesion through integrins. Cell 1991; 65 : 859-73
38. Von Adrian UH, Chambers JD, McFvoy LM, et al. Two-stcp model of lcukocyte-endothclial ccll interaction in inflammation : distinct roles for LECAM-1 and the lcukocyte $\beta 2$ integrins in vivo. Proc Natl Acad Sci USA 1991 ; 88 : 7538-42.

\section{Summary}

Selectins and their role in leucocyte adhesion to endothelial cells

A recently identified gene encodes three adhesion molecules involved in leucocyte-endothelial cell interactions. These " selectins " share a high degree of sequence homology and particularly a calcium-type lectin domain, which suggests their ligand is an oligosaccharide. The P- and E-selectins are only expressed on the surface of stimulated platelets and endothelial cells and interact with neutrophils and monocytes. They have the same ligand : sialyl Lewis ${ }^{\times}\left(\mathrm{SLe}^{\times}\right)$. Lselectin, which is expressed on lymphocytes, participates in their attachment to high endothelial cells of peripheral lymph nodes via a glycoprotein, the oligosaccharide determinant of which could be SLe $^{\times}$. The selectins are involved in the first step of leucocyte binding to, and extravasation through, endothelium during an inflammatory response. Oligosaccharide drugs could be designed to block adhesive interactions in inflammatory disorders.
39. Winkelhake JL. Will complex carbohydrate ligands of vascular selectins be the next gencration of non steroidal antiinflammatory drugs? Glycoconjugate J 1991 ; $8: 381-6$

L'ASSIM, Association des Enseignants d'Immunologic des Universités de Langue française s'est constituée en 1973. A l'époque l'Immunologie devenait une science enfin reconnue comme discipline par l'Université française et ce à partir d'enseignants issus de l'entité désignée à l'époque sous le vocable " microbiologie ". Les fondateurs de l'Association, appelée à l'époque Amicale, ont voulu très rapidement qu'un manuel soit mis à disposition des Étudiants du second cycle, tant au niveau des études médicales que des études pharmaceutiques. C'est ainsi qu'est né "Ig Cordelier". Désormais l'ASSIM, qui a rédigé plusieurs livres: Immunologie Générale, Immunologie Clinique et Technique en Immunologie, se propose, par des réunions pédagogiques, par des colloques, des séminaires, de rassembler tous les enseignants de l'immunologie et ce à partir du secondaire. Désormais, en cohésion avec la SFI (Société Française d'Immunologie) elle a pour finalité, la défense de la langue française et au-delà du transfert des concepts immunologiques dans notre langue. Mais, il est exclu qu'elle considère le français comme langage scientifique universel... 